\title{
Utility of Magnetic Resonance Imaging (MRI) and It's Comparison with Electroencephalogram (EEG) in First Onset Seizures in Children
}

\author{
Mohan B. ${ }^{1}$, Ranoji Mane ${ }^{2}$, Karthik G A ${ }^{3}$, Vimal Raj ${ }^{4}$, Parthasarathy ${ }^{5}$, Venkatramana Bhat ${ }^{6}$ \\ ${ }^{1}$ Consultant Radiologist, ${ }^{2}$ Assistant Professor, ${ }^{3}$ Consultant Radiologist, ${ }^{4}$ Consultant Radiologist, ${ }^{5}$ Professor, ${ }^{6}$ Professor and \\ HOD, Gulbarga Institute of Medical Sciences, Narayana Hrudayalaya Institute of Medical Sciences, Bangalore, Karnataka, \\ India
}

Corresponding author: Dr. Ranoji Mane, \#8-1545/27, Shivaji Building, Shivaji Nagar, Humnabad Road, Kalaburgi -585104, Karnataka, India

DOI: 10.21276/ijcmsr.2018.3.2.2

I

How to cite this article: Utility of magnetic resonance imaging (MRI) and it's comparison with electroencephalogram (EEG) in First onset seizures in children. Mohan B., Ranoji Mane, Karthik G A, Vimal Raj, Parthasarathy, Venkatramana Bhat. International Journal of Contemporary Medicine Surgery and Radiology. 2018;3(2):B4-B7.

\section{A B S T R A C T}

Introduction: Seizures are symptoms of abnormal brain function and occur due to diverse etiology. The aim of the study was to evaluate the diagnostic efficacy of MRI of the brain in children with first onset seizures and to compare the diagnostic yields of MRI and EEG individually and in combination.

Material and methods: The study consists of One hundred and twelve patients (112) with an age range from 5 days to 18 years with first onset seizures who underwent MR imaging and EEG. Sensitivity was calculated to both MRI and EEG separately and together. Chi square test was applied. 95\% confidence interval of sensitivity was calculated. Epi info version 3 was used for statistical calculations.

Results: Out of 112 cases, 69\% were male and 31\% female. Out of 112 cases, 50 cases (45\%) showed epileptiform abnormalities on MRI, most common being infection and inflammation (26\%) followed by HIE sequelae (24\%). Out of 112 cases, 40 (36\%) showed focal epileptiform abnormalities on EEG. Out of 112 cases, $15(13 \%)$ were both MRI positive and EEG positive, $37(33 \%)$ were both MRI and EEG negative, $35(31 \%)$ were only MRI positive and $25(22 \%)$ were only EEG positive. Conclusion: MRI and EEG are the methods of choice for evaluation of patients with seizure, MRI can identify structural brain lesions, that can serve as epileptogenic foci, that might be surgically resectable if the patient becomes refractory to medical treatment.

Key Words: Epilepsy, Magnetic Resonance Imaging.

\section{INTRODUCTION}

Seizures are symptoms of abnormal brain function and occur due to diverse etiology. Prompt management may reduce the chance of a second seizure attack by as much as $50 \%{ }^{1}$ Epilepsy is a chronic condition characterized by recurrent seizures provoked by an acute systemic or neurologic insult. An epileptic seizure is a clinical manifestation of abnormal, excessive neuronal activity arising in the grey matter of the cerebral cortex.

Magnetic Resonance Imaging (MRI) is the current imaging tool of choice in the investigation of patients with seizures. ${ }^{2}$ MRI identifies structural lesions that require urgent treatment such as high-grade gliomas, arteriovenous malformations, infections and malformations of cortical development. ${ }^{3}$

The International League against Epilepsy (ILAE) Subcommittee for Pediatric Neuroimaging examined the usefulness of, and the indications for, neuroimaging in the evaluation of children with newly diagnosed epilepsy. ${ }^{4,5}$
Nearly, $50 \%$ of individual imaging studies in children with new-onset seizures were reported to be abnormal.15-20\% of imaging studies provided useful information on etiology or seizure focus and $2-4 \%$ provided information that potentially altered immediate medical management. ${ }^{5,6,7,8}$

This study aimed to evaluate the diagnostic efficacy of MRI of the brain in children with first onset seizures and to compare the diagnostic yields of MRI and EEG individually and in combination.

\section{MATERIAL AND METHODS}

The study consists of One hundred and twelve patients (112) with an age range from 5 days to 18 years with first onset seizures underwent MR imaging and EEG. The study was started after the approval from the Ethical Committee. Written informed consent was obtained from all the participants. The study was done in Narayana Hrudayalaya Hospital from November 2013 to October 2014. 
MRI Protocol: Images were acquired on 1.5 Tesla (PHILPS ACHIEVA or GE SIGNA HdXT) using a head coil and gradient strength of $45 \mathrm{mT}$. The protocol consists of axial, Sagittal T1 weighted sequence (T1W), and axial T2 weighted sequence (T2W), axial Fluid attenuation inversion recovery sequence (FLAIR), axial Gradient echo (GRE) and Diffusion weighted imaging (DWI).

Sample size of patients: The sample size was estimated using nMaster software version 2.0. Assuming the proportion of individuals with MRI positivity of $72 \%$, with relative precision of $12 \%$ and desired confidence interval of $95 \%$, sample size requirement was determined to be 104 cases.

\section{Inclusion criteria}

All patients aged below 18 years presenting with first onset seizures.

\section{Exclusion criteria}

1. Any previously diagnosed non-central nervous system disorders liable to cause seizures.

2. Patients with known contraindications to MRI.

3. Syncopal and hypoglycemic attacks, pseudo-seizures or drug induced seizures.

4. Patients presenting with head injury.

\section{STATISTICAL ANALYSIS}

Quantitative variables were expressed in terms of mean, standard deviation or median interquartile range with confidence interval of $95 \%$. The entire qualitative variable was expressed in terms of proportion. Chi square test of significance $(p<0.005)$ was used to test for the difference in proportion. The correlation between MRI brain and EEG was studied using Mc Nemer test.

\begin{tabular}{|l|c|c|}
\hline MRI epileptogenic lesions & $\begin{array}{c}\text { Number } \\
\text { of patients } \\
\text { (n=50) }\end{array}$ & $\%$ \\
\hline $\begin{array}{l}\text { Hypoxic Ischemic Encephalopathy } \\
\text { Sequelae }\end{array}$ & 12 & 24 \\
\hline Infection and Inflammation & 13 & 26 \\
\hline Hippocampal sclerosis & 3 & 6 \\
\hline Acute Infarct & 3 & 6 \\
\hline Chronic Infarct & 4 & 8 \\
\hline Tumor and tumor like lesions & 4 & 8 \\
\hline Metabolic disorder & 3 & 6 \\
\hline Vascular Malformation & 1 & 2 \\
\hline PRES & 2 & 4 \\
\hline Hypomyelination & 3 & 6 \\
\hline Vasculitis & 2 & 4 \\
\hline Total & 50 & 100 \\
\hline \multicolumn{2}{|c|}{} \\
\hline
\end{tabular}

\begin{tabular}{|l|l|l|l|}
\hline \multirow{2}{*}{ MRI } & EEG & \multirow{2}{*}{ Total } \\
\cline { 2 - 3 } & Normal & Abnormal & \\
\hline Normal & $37(33.1 \%)$ & $25(22.3 \%)$ & $62(55.4 \%)$ \\
\hline Abnormal & $35(31.2 \%)$ & $15(13.4 \%)$ & $50(44.6 \%)$ \\
\hline Total & $72(64.3 \%)$ & $40(35.7 \%)$ & $112(100 \%)$ \\
\hline \multicolumn{3}{|c|}{ Table-2: MRI and EEG correlation } \\
\hline
\end{tabular}

\begin{tabular}{|l|l|l|l|}
\hline A) Sensitivity test: \\
\hline & Positive & Negative & $\begin{array}{l}\text { Sensitivity (95\% } \\
\text { confidence interval) }\end{array}$ \\
\hline MRI & 50 & 62 & $44.64 \%(35.77,53.88)$ \\
\hline EEG & 40 & 72 & $35.71 \%(27.45,44.93)$ \\
\hline MRI + EEG & 75 & 37 & $66.96 \%(57.38,75.53)$ \\
\hline B) Chi-square test for comparison of results of MRI and EEG \\
\hline MRI & \multicolumn{4}{|c|}{ EEG } \\
\cline { 2 - 4 } & Normal & Abnormal & \\
\hline Normal & $37(33.1 \%)$ & $25(22.3 \%)$ & $62(55.4 \%)$ \\
\hline Abnormal & $35(31.2 \%)$ & $15(13.4 \%)$ & $50(44.6 \%)$ \\
\hline Total & $72(64.3 \%)$ & $40(35.7 \%)$ & $112(100 \%)$ \\
\hline $\begin{array}{l}\text { Chi- square is 1.285 and p-value 0.12 which shows no signifi- } \\
\text { cant difference between MRI and EEG }\end{array}$ \\
\hline \multicolumn{5}{|c|}{ Table-3: Statistical analysis } \\
\hline
\end{tabular}

\section{RESULTS}

In this study 50 patients (45\%) had positive findings on MRI, the most common epileptogenic lesions, were infection and inflammation seen in 13 patients(26\%) followed by hypoxic ischemic encephalopathy sequelae in 12 patients (24\%). Other causes were acute, chronic infarcts, metabolic disorders and vascular malformations (Table 1).

There were 15 patients (13\%) with an abnormality on both MRI and EEG. So, abnormal MRI and EEG were concordant in $13 \%$ of subjects in this study. There were 37 patients (33\%) where both MRI and EEG were normal (Table 2).

The sensitivity (95\% Confidence Interval) for MRI is $44.64 \%$ and EEG is $35.71 \%$, combined sensitivity for MRI and EEG is $66.96 \%$ (Table $3 \mathrm{~A}$ ).

However there is no statistical significant difference between MRI and EEG as shown in the Chi-square test with $\mathrm{p}$-value of 0.12 (Table 3B).

\section{DISCUSSION}

The primary goal in evaluating a patient's first seizure is to establish whether the seizure resulted from a systemic process or intrinsic focus from the central nervous system, if found to be focal, to demonstrate underlying brain pathology.

Neuroimaging is central to the evaluation of patients with first onset seizure, especially in the identification of a structural brain lesion that can be the epileptogenic focus. ${ }^{9}$ This study evaluated 112 patients who presented with a clinical diagnosis of first onset seizure as per criteria laid by ILAE 1981.

The clinical history of the patient was recorded and was evaluated with EEG and MRI of brain. All 112 patients underwent EEG and MRI of brain.

Patient characteristics: Age range of our patients was between 5 days to 18 years with a mean age of 7.4 years. The number of male patients were 77 (69\%) and female patients 35 (31\%).

About $74 \%$ of patients presented with generalized tonic clonic seizures and $15 \%$ presented with complex partial seizures.

50 patients (45\%) had positive findings on MRI. Reported 
incidence of detection of epileptogenic lesions using only MRI varies between $14 \%$ and $48 \%{ }^{10}$

In this study, among the potentially epileptogenic lesions, infection and inflammation were the most common, seen in 13 subjects (26\%) followed by hypoxic ischemic encephalopathy sequelae. Of the patients in infection and inflammation group, eight patients had granulomatous lesions and five patients had non granulomatous infection. Out of the 8 patients with granulomatous lesions, 4 patients had neurocysticercosis, 3 had tuberculoma and 1 patient had an active granulomatous lesion which could not be specifically categorized to either neurocysticerosis or tuberculous etiology. ${ }^{11,12}$

Del Brutto et al in 2012 concluded that cysticercosis is the leading cause of acquired epilepsy worldwide and the main reason for a higher prevalence of epilepsy in developing countries. ${ }^{13,14}$

In this study, of 50 potentially epileptogenic lesions, hippocampal sclerosis was an infrequent finding seen in 3 subjects (6\%). Previous studies all the age groups have reported the incidence of mesial temporal sclerosis varying from $8 \%$ to $30 \%$.

Tumours were relatively uncommon (8\%) of which majority were extraxial tumours with significant compression on brain parenchyma. Only one patient had an intra axial lesion.

Hypomyelination was seen in 3 patients (6\%) which is an entity seen only in pediatric population.

Acute infarcts were seen in 3 patients who presented with first onset seizure. Out of these 3 patients, 2 patients also had acute venous sinus thrombosis.

Chronic infarct with gliotic changes and posterior reversible encephalopathy syndrome (PRES) were seen in 4 and 2 patients respectively. Both the subjects with PRES were in the higher age group of 11-18 years (Mean 15.5 years).

Patients who presented with complex partial seizures (15) had a higher proportion of potentially epileptogenic lesions (65\%), compared to the patients with generalized tonic clonic seizures, which is comparable to the study done by Hakami T et al. ${ }^{15}$

Partial onset seizures that secondarily generalize rapidly can be misinterpreted as primary generalized seizures. If, however, the MRI shows a structural lesion that is the likely source of the seizures, then they can be classified as partial. ${ }^{16}$ Among the nonepileptogenic abnormalities detected on MRI, non-specific white matter hyper intensities was the most frequent finding followed by mild cerebral atrophy. Hence these subjects were not included under the MRI positive group.

\section{Electroencephalography (EEG)}

Of the 112 patients who underwent EEG, 40 patients (36\%) showed focal epileptiform abnormalities and 72 patients (65\%) showed normal recordings which included generalized non specific waves and background beta and delta waves which could not be attributed to the seizures. The diagnostic yield of EEG in subjects presenting with first onset seizure was $36 \%$ in this study.

In patients with first onset seizure, Hakami $\mathrm{T}$ et al showed EEG abnormality in $31 \%$ subjects. ${ }^{15,17,18}$

This study showed abnormal EEG recordings in $6(35 \%)$ out of 17 patients who presented with complex partial seizures and $30(36 \%)$ out of 83 patients who presented with generalized tonic clonic seizures.

\section{MRI and EEG correlation}

There were 15 patients (13\%) with an abnormality on both MRI and EEG. So, abnormal MRI and EEG were concordant in $13 \%$ of subjects in this study, which is similar to another study. ${ }^{15}$ There were 37 patients (33\%) where both MRI and EEG were normal.

There were 35 patients (31\%) with an abnormal MRI but normal EEG. This study shows that EEG was normal in $31 \%$ of patients with epileptogenic lesions on MRI which is similar to study done by Hakami $\mathrm{T}$ et al ${ }^{15}$ where EEG was normal in $55 \%$ of patients with positive MRI findings.

\section{Limitations of the study}

- Histopathological diagnosis was not done in all the cases of space occupying lesions (granulomatous lesions and tumours).

- CSF analysis was not done in all cases of meningitis and meningo-encephalitis. Diagnosis of these cases was made on imaging appearances only.

\section{CONCLUSION}

MRI and EEG are the vital diagnostic tools for accurate diagnosis in a patient presenting with first episode of seizure. Along with a careful history and clinical examination, they allow definition of the epilepsy syndrome in two-thirds of these patients and help assess the individual risk for seizure recurrence.

MRI is central to the evaluation of patients with seizure, especially in the identification of structural brain lesions that could be the epileptogenic foci. Information obtained may help to decide medical or surgical management strategy.

We recommend that all patients with first-onset seizure should have an MRI study along with EEG.

\section{REFERENCES}

1. Susan T, Herman MD. Single Unprovoked Seizures. Current Treatment Options in Neurology 2004; 6(4): 243-55.

2. Scott N. Atlas. Magnetic Resonance Imaging of the brain and spine. 4th edition, p. 2-14, 307-339.

3. Sachin Rastogi, Christopher Lee, Noriko Salamon. Neuroimaging in pediatric epilepsy: A multimodality approach. Radio Graphics 2008;28(2):1079-1095.

4. Fisher RS et al. Epileptic seizures and epilepsy: definitions proposed by the International League Against Epilepsy (ILAE) and the International Bureau for Epilepsy (IBE).Epilepsia. 2005;46(4):470-2.

5. The International League Against Epilepsy, Committee for Neuroimaging, Subcommittee for Pediatric. Guidelines for Imaging Infants and Children with Recent-Onset Epilepsy. Epilepsia 2009;50(9):2147-53.

6. The Commission on classification and terminology of the ILAE. Proposal for revised classification of epilepsy and epileptic syndromes. Epilepsia 1989;30(4):389-399.

7. Berg AT et al. Revised terminology and concepts for organization of seizures and epilepsies: report of the ILAE Commission on Classification and Terminology, 
2005-2009. Epilepsia 2010;51(3):676.

8. Wieser HG, ILAE Commission on Neurosurgery of Epilepsy (2004) ILAE commission report: mesial temporal lobe epilepsy with hippocampal sclerosis. Epilepsia 2004;45(5):695-714.

9. Bronen RA, Fullbright RR, Spencer DD, et al. Refractory epilepsy: comparison of MR imaging $\mathrm{CT}$ and histopathological findings in 117 patients. Radiology 1996;201(6):97-105.

10. Liu RS, Lemieux L, Bell GS, et al. The structural consequences of newly diagnosed seizures. Ann Neurol 2002;52(4):573-580.

11. Price HI, Danziger A. Computed tomography in cranial tuberculosis. AJR 1978;130(2):769-771.

12. Salgado P, OH Del Brutoo, O.Talamas, MA Zenteno and J. Rodrisuez - Carbajal. Intracranial tuberculoma MR imaging. Neurology 1989;31(1):299-302.

13. Del Brutto OH. Neurocysticercosis: a review. Scientific World J. 2012:159821.

14. Sanchetee PC, Venkataraman CS, Dhamija RM, Roy AK. Epilepsy as a manifestation of neurocysticercosis. J Assoc Physician India 1991;39(5):325-8.

15. Hakami $T$ et al. MRI - identified pathology in adults with new-onset seizures. Neurology 2013;81(7):920927.

16. Betting LE et al. MRI reveals structural abnormalities in patients with idiopathic generalized epilepsy. Neurology 2006;67(1):848.

17. Gilbert DL, Sethuraman G, Kotagal U, Buncher CR. Meta-analysis of EEG test performance shows wide variation among studies. Neurology 2003;60(3):564.

18. Seneviratne U, Cook M, D'Souza W. The electroencephalogram of idiopathic generalized epilepsy. Epilepsia 2012;53(5):234.

Source of Support: Nil; Conflict of Interest: None

Submitted: 20-03-2018; Published online: 25-04-2018 